Research Paper

\title{
Appraisal of Prognostic Interaction between Sidedness and Mucinous Histology in Colon Cancer: A Population-Based Study Using Inverse Probability Propensity Score Weighting
}

\author{
Zi-Xian Wang ${ }^{1 *}$, Lu-Ping Yang $12^{*}$, Hao-Xiang Wu ${ }^{1,2^{*}}$, Dong-Dong Yang ${ }^{1^{*}}$, Pei-Rong Ding 3 , Dan Xie ${ }^{4}$, Gong \\ $\mathrm{Chen}^{3 \#}$, Yu-Hong $\mathrm{Li}^{1 \#}$, Feng Wang1\#, Rui-Hua $\mathrm{Xu}^{1 \# \bowtie}$ \\ 1. Department of Medical Oncology, Sun Yat-Sen University Cancer Center, State Key Laboratory of Oncology in South China, Collaborative Innovation \\ Center for Cancer Medicine, Guangzhou, 510060, China \\ 2. Faculty of Medical Sciences, Sun Yat-Sen University, Guangzhou, 510080, China \\ 3. Department of Colorectal Surgery, Sun Yat-Sen University Cancer Center, State Key Laboratory of Oncology in South China, Collaborative Innovation \\ Center for Cancer Medicine, Guangzhou, 510060, China \\ 4. Department of Pathology, Sun Yat-Sen University Cancer Center, State Key Laboratory of Oncology in South China, Collaborative Innovation Center for \\ Cancer Medicine, Guangzhou, 510060, China \\ ${ }^{*}$ Authors contributed equally to this study. \\ \#Joint senior authors
}

$\square$ Corresponding author: Prof. Rui-Hua Xu, Department of Medical Oncology, Sun Yat-Sen University Cancer Center, State Key Laboratory of Oncology in South China, Collaborative Innovation Center for Cancer Medicine, 651 Dong Feng Road East, Guangzhou 510060, China. Tel and Fax: +86-20-8734 3468; E-mail: xurh@sysucc.org.cn

( ) Ivyspring International Publisher. This is an open access article distributed under the terms of the Creative Commons Attribution (CC BY-NC) license (https://creativecommons.org/licenses/by-nc/4.0/). See http://ivyspring.com/terms for full terms and conditions.

Received: 2018.06.20; Accepted: 2018.10.03; Published: 2019.01.01

\begin{abstract}
Introduction: Colon cancer with different sidedness (right vs. left) and histology (mucinous vs. non-mucinous) may represent different disease entities. We investigated whether the prognostic values of sidedness and histology differed according to each other.

Materials and Methods: We analyzed 81342 patients with stage II-IV colon cancer from the Surveillance, Epidemiology, and End Results database between 2004 and 2012. Patients were divided into four subgroups on the basis of sidedness and histology: non-mucinous right-sided, non-mucinous left-sided, mucinous right-sided, and mucinous left-sided subgroups. Among each tumor stage, median overall survival (mOS) was compared between these subgroups after inverse probability propensity score weighting to handle confounding factors.

Results: In the stage IV subgroup, the prognosis for non-mucinous left-sided tumors (weighted mOS, 24.5 months) was significantly better than that for non-mucinous right-sided tumors (weighted mOS, 16.5 months; $P<0.001$ ) and that for mucinous left-sided tumors (weighted mOS, 16.5 months; $P<0.001$ ), whereas the survival was similar between left-sided and right-sided tumors with the mucinous subtype (weighted mOS, 16.5 months for both; $P=0.570$; test for interaction between sidedness and histology, $P_{\text {interaction }}<0.001$ ), and between mucinous and non-mucinous tumors in the right-sided colon (weighted mOS, 16.5 months for both; $P=0.207$ ). Similar findings were detected in the stage III subgroup $\left(P_{\text {interaction }}<0.001\right)$. In the stage II subgroup, the survival was comparable among the four sidedness-histology subgroups $\left(P=0.159\right.$ and $\left.P_{\text {interaction }}=0.466\right)$.

Conclusions: In stage III/IV colon cancer, the prognostic value of sidedness differed according to histology, and vice versa. By contrast, neither should be considered in risk stratification for stage II colon cancer.
\end{abstract}

Key words: colon cancer, tumor side, mucinous histology, survival, surveillance, epidemiology, end results

\section{Introduction}

Colorectal cancer is one of the most common malignancies and among the leading causes of cancer-related deaths worldwide. [1] Researchers have recently focused on analyzing the diversity of molecular profiles within the large intestine; [2] there is also renewed interest in clinicopathologic features as prognostic and predictive indicators, such as side 
(i.e., right vs. left) of the primary tumor [3] and histologic type (i.e., mucinous vs. non-mucinous). [4]

Previous studies have shown that compared to patients with left-sided colon cancer (LSCC), those with right-sided colon cancer (RSCC) tend to be older and of the female gender, and have poorly differentiated and advanced stage tumors. [5-7] Additionally, RSCC exhibits a different molecular profile from LSCC. [8-10] However, data comparing prognosis between RSCC and LSCC are conflicting, particularly when patients are stratified by tumor stage. $[3,6,7,11,12]$

Mucinous adenocarcinoma (MAC) is a tumor comprising more than 50\% extracellular mucin. [13] MAC is more often correlated with right sidedness, advanced stage at presentation, and peritoneal dissemination, and has different molecular biological patterns than non-mucinous adenocarcinoma (NMAC). [4, 14-16] However, the prognostic value of the mucinous histology remains controversial. Several studies reported that MAC was associated with poor prognosis, [16-18] while others did not confirm this conclusion. [4, 19-21]

To our knowledge, data are scarce regarding the prognostic impact of sidedness and histology stratified by each other. We suspect that non-mucinous RSCC, non-mucinous LSCC, mucinous RSCC, and mucinous LSCC might represent different disease entities. In the present population-based study of patients with stage II-IV colon cancer, we aim to investigate whether the prognostic impacts of tumor side and histology on patient survival differ according to each other.

\section{Materials and Methods}

\section{Study cohort}

Using the National Cancer Institute's Surveillance, Epidemiology, and End Results (SEER) database (18 registries), we identified 254040 patients with colon cancer diagnosed between January 2004 and December 2012. As presented in Figure 1, 81342 patients met the inclusion criteria for this study. Because SEER is public-use data, no institutional review was sought and informed consent was waived.

Overall survival (OS) was the primary outcome of interest. The examined covariates included race, age, sex, marital status, year of diagnosis, SEER region, tumor grade, tumor location, tumor histologic type, $\mathrm{T}$ and $\mathrm{N}$ category ( $6^{\text {th }}$ edition), and the total number of lymph nodes evaluated.

Patients with cancer of the cecum, ascending colon, and transverse colon were defined as having RSCC, and patients with cancer of the descending or sigmoid colon were considered as having LSCC.
NMAC was defined on the basis of the third edition of the International Classification of Diseases for Oncology histology codes 8140, 8144, 8210, 8211, 8221, 8261,8262 , and 8263 and MAC, on the basis of the codes 8480 and 8481 . Patients were further divided into four subgroups on the basis of sidedness and histology (S-H): non-mucinous RSCC, non-mucinous LSCC, mucinous RSCC, and mucinous LSCC.

This study is based on SEER database which is public-use data, thus no institutional review was sought and informed consent was waived.

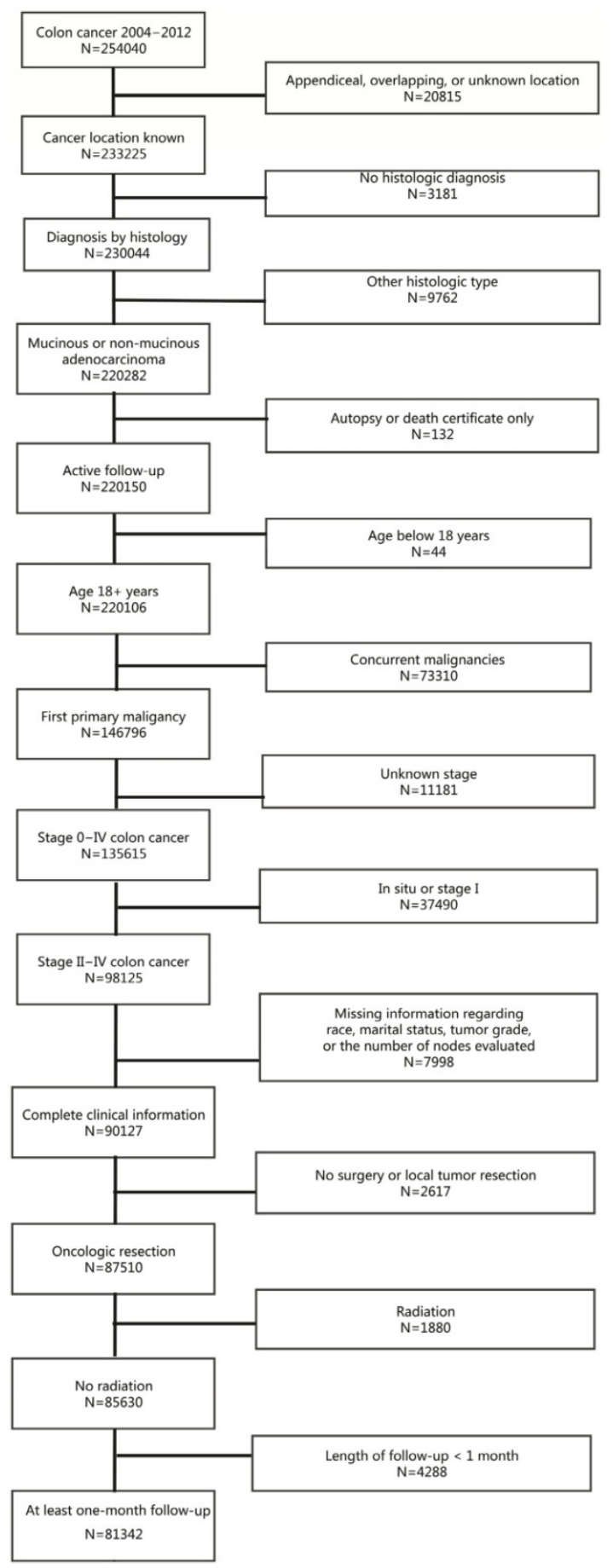

Figure 1. Flowchart of patient selection process for the study cohort. 


\section{Statistical analysis}

The $\chi^{2}$ test was used to compare baseline clinicopathologic features between the $\mathrm{S}-\mathrm{H}$ groups. To allow for more accurate measurement of the prognostic impact of sidedness and histology, we used an inverse probability propensity score weighting approach for multiple groups $(\geq 2)$ [22] with stratification for tumor stage to generate a weighted cohort in which the clinicopathologic features (i.e., age, sex, race, marital status, year of diagnosis, SEER region, tumor grade, $\mathrm{T}$ and $\mathrm{N}$ stage, and total evaluated lymph node count) were balanced between each pair of S-H groups. Propensity score methods are useful to reduce or minimize the effects of confounding when estimating the effects of treatments, exposures, or interventions in observational or non-randomized interventional studies. [23] It has been shown that that both the inverse propensity score weighting and propensity score matching allow for the estimation of the causal effect with minimal bias. [24] As compared with the propensity score matching approach, inverse propensity score weighting can be more flexibly applied to comparison between multiple groups and hence was used in the current study. [22, 25]

After inverse propensity score weighting, OS was compared between the RSCC and LSCC groups and between the MAC and NMAC groups, as well as among the S-H groups. Survival curves for patients in different groups were generated using the Kaplan-Meier method and compared using the log-rank test. Cox proportional hazards models with inverse propensity score weighting and robust sandwich variance estimators [26] were used to assess the associations of sidedness, histology, and S-H group with hazard ratios (HRs) for death. Interaction tests were performed to determine whether the impacts of sidedness and histology on OS were significantly influenced by each other using Cox proportional hazards models.

Statistical significance was set as $P<0.050$ in a two-tailed test. Statistical analyses were performed using SAS v. 9.3 (SAS Institute, Cary, NC, USA), IBM SPSS Statistics for Windows v. 19.0 (IBM Corp., Armonk, NY, USA), and R v. 3.4.1 (http://www.rproject.org).

\section{Results}

\section{Patient characteristics}

Table 1 summarizes the patient characteristics of the study cohort (81342 cases). Overall, 49,398 (60.7\%) patients had RSCC and 31,944 (39.3\%) patients had LSCC, 9265 (11.4\%) patients with MAC while 72077 $(88.6 \%)$ patients with NMAC. The non-mucinous
RSCC, non-mucinous LSCC, mucinous RSCC, and mucinous LSCC subgroups included 42395 (52.1\%), $29682(36.5 \%), 7003(8.6 \%)$, and $2262(2.8 \%)$ patients, respectively. The mean follow-up duration was $35.7 \pm$ 29.3 months.

Table 1. Clinicopathologic characteristics of the study cohort of patients with stage II-IV colon cancer $(\mathrm{N}=81342)$

\begin{tabular}{|c|c|}
\hline Variable & Number (\%) \\
\hline \multicolumn{2}{|l|}{ Tumor side } \\
\hline Right & $49398(60.7 \%)$ \\
\hline Left & $31944(39.3 \%)$ \\
\hline \multicolumn{2}{|l|}{ Mucinous histology } \\
\hline Yes & $9265(11.4 \%)$ \\
\hline No & $72077(88.6 \%)$ \\
\hline \multicolumn{2}{|l|}{ S-H group } \\
\hline Non-mucinous RSCC & 42395 (52.1\%) \\
\hline Non-mucinous LSCC & $29682(36.5 \%)$ \\
\hline Mucinous RSCC & $7003(8.6 \%)$ \\
\hline Mucinous LSCC & $2262(2.8 \%)$ \\
\hline \multicolumn{2}{|l|}{ Age, years } \\
\hline$<50$ & $9153(11.3 \%)$ \\
\hline $50-59$ & $15133(18.6 \%)$ \\
\hline $60-69$ & $19170(23.6 \%)$ \\
\hline $70-79$ & $20017(24.6 \%)$ \\
\hline$\geq 80$ & $17869(22.0 \%)$ \\
\hline \multicolumn{2}{|l|}{ Sex } \\
\hline Male & $38748(47.6 \%)$ \\
\hline Female & $42594(52.4 \%)$ \\
\hline \multicolumn{2}{|l|}{ Race } \\
\hline Non-Hispanic white & $56190(69.1 \%)$ \\
\hline Non-Hispanic black & $10079(12.4 \%)$ \\
\hline Hispanic & $8047(9.9 \%)$ \\
\hline Other & $7026(8.6 \%)$ \\
\hline \multicolumn{2}{|l|}{ Marital status } \\
\hline Married & $45364(55.8 \%)$ \\
\hline Single/other & $20034(24.6 \%)$ \\
\hline Widowed & $15944(19.6 \%)$ \\
\hline \multicolumn{2}{|l|}{ Year of diagnosis } \\
\hline $2004-2006$ & $27968(34.4 \%)$ \\
\hline 2007-2009 & $27785(34.2 \%)$ \\
\hline 2010-2012 & $25589(31.5 \%)$ \\
\hline \multicolumn{2}{|l|}{ SEER region } \\
\hline Midwest & $13160(16.2 \%)$ \\
\hline Northeast & $12780(15.7 \%)$ \\
\hline South & $15823(19.5 \%)$ \\
\hline West & $39579(48.7 \%)$ \\
\hline \multicolumn{2}{|l|}{ Tumor grade } \\
\hline G1/G2 & $62334(76.6 \%)$ \\
\hline G3/G4 & $19008(23.4 \%)$ \\
\hline \multicolumn{2}{|l|}{ T stage } \\
\hline T1 & $1521(1.9 \%)$ \\
\hline T2 & $3226(4.0 \%)$ \\
\hline $\mathrm{T} 3$ & $60978(75.0 \%)$ \\
\hline $\mathrm{T} 4$ & $15617(19.2 \%)$ \\
\hline \multicolumn{2}{|l|}{$\mathrm{N}$ stage } \\
\hline No & $36044(44.3 \%)$ \\
\hline N1 & $26624(32.7 \%)$ \\
\hline N2 & $18674(23.0 \%)$ \\
\hline \multicolumn{2}{|l|}{ AJCC stage } \\
\hline II & 33414 (41.1) \\
\hline III & $32480(39.9)$ \\
\hline IV & $15448(19.0)$ \\
\hline \multicolumn{2}{|c|}{ Total evaluated node count } \\
\hline$<12$ & $19635(24.1 \%)$ \\
\hline$\geq 12$ & $61707(75.9 \%)$ \\
\hline
\end{tabular}

RSCC, right-sided colon cancer; LSCC, left-sided colon cancer; SEER, Surveillance, Epidemiology, and End Results; AJCC, American Joint Committee on Cancer 
Table 2. Patient characteristics of the study cohort stratified by tumor side and histology

\begin{tabular}{|c|c|c|c|c|c|c|}
\hline Variable & $\begin{array}{l}\text { RSCC } \\
(\%)\end{array}$ & $\begin{array}{l}\text { LSCC } \\
(\%)\end{array}$ & $P$ value & $\begin{array}{l}\text { NMAC } \\
(\%)\end{array}$ & $\begin{array}{l}\text { MAC } \\
(\%)\end{array}$ & $P$ value \\
\hline Mucinous histology & & & $<0.001$ & & & - \\
\hline Yes & 14.2 & 7.1 & & - & - & \\
\hline No & 85.8 & 92.9 & & - & - & \\
\hline Age, years & & & $<0.001$ & & & $<0.001$ \\
\hline$<50$ & 8.6 & 15.3 & & 11.6 & 11.2 & \\
\hline $50-59$ & 15.6 & 23.3 & & 16.3 & 18.9 & \\
\hline $60-69$ & 22.9 & 24.7 & & 22.3 & 23.7 & \\
\hline $70-79$ & 26.7 & 21.4 & & 25.4 & 24.5 & \\
\hline$\geq 80$ & 26.3 & 15.3 & & 24.3 & 21.7 & \\
\hline Sex & & & $<0.001$ & & & 0.012 \\
\hline Male & 44.7 & 52.2 & & 46.4 & 47.8 & \\
\hline Female & 55.3 & 47.8 & & 53.6 & 52.2 & \\
\hline Race & & & $<0.001$ & & & $<0.001$ \\
\hline Non-Hispanic white & 71.2 & 65.7 & & 71.9 & 68.7 & \\
\hline Non-Hispanic black & 12.6 & 12.0 & & 11.6 & 12.5 & \\
\hline Hispanic & 9.3 & 10.9 & & 10.1 & 9.9 & \\
\hline Other & 6.9 & 11.4 & & 6.3 & 8.9 & \\
\hline Marital status & & & $<0.001$ & & & $<0.001$ \\
\hline Married & 54.2 & 58.2 & & 54.3 & 56.0 & \\
\hline Widowed & 22.5 & 15.1 & & 21.7 & 19.3 & \\
\hline Single/other & 23.3 & 26.7 & & 24.0 & 24.7 & \\
\hline Year of diagnosis & & & 0.154 & & & $<0.001$ \\
\hline 2004-2006 & 34.2 & 34.7 & & 39.4 & 33.7 & \\
\hline 2007-2009 & 34.1 & 34.2 & & 31.9 & 34.4 & \\
\hline 2010-2012 & 31.7 & 31.1 & & 28.7 & 31.8 & \\
\hline SEER region & & & $<0.001$ & & & $<0.001$ \\
\hline Midwest & 17.0 & 14.9 & & 17.3 & 16.0 & \\
\hline Northeast & 16.0 & 15.2 & & 18.4 & 15.4 & \\
\hline South & 18.8 & 20.5 & & 17.1 & 19.8 & \\
\hline West & 48.2 & 49.3 & & 47.2 & 48.8 & \\
\hline Tumor grade & & & $<0.001$ & & & $<0.001$ \\
\hline G1/G2 & 72.6 & 82.9 & & 74.9 & 76.9 & \\
\hline G3/G4 & 27.4 & 17.1 & & 25.1 & 23.1 & \\
\hline T stage & & & $<0.001$ & & & $<0.001$ \\
\hline $\mathrm{T} 1$ & 1.3 & 2.7 & & 0.8 & 2.0 & \\
\hline $\mathrm{T} 2$ & 3.6 & 4.5 & & 2.9 & 4.1 & \\
\hline T3 & 75.6 & 74.0 & & 71.1 & 75.5 & \\
\hline $\mathrm{T} 4$ & 19.4 & 18.9 & & 25.2 & 18.4 & \\
\hline N stage & & & $<0.001$ & & & $<0.001$ \\
\hline No & 45.9 & 41.9 & & 46.7 & 44.0 & \\
\hline N1 & 30.8 & 35.7 & & 28.7 & 33.2 & \\
\hline N2 & 23.3 & 22.4 & & 24.6 & 22.7 & \\
\hline AJCC stage & & & $<0.001$ & & & $<0.001$ \\
\hline II & 43.3 & 37.3 & & 43.8 & 40.7 & \\
\hline III & 38.8 & 41.6 & & 37.5 & 40.2 & \\
\hline IV & 17.9 & 20.7 & & 18.8 & 19.0 & \\
\hline \multicolumn{2}{|c|}{ Total evaluated node count } & & $<0.001$ & & & 0.002 \\
\hline$<12$ & 20.0 & 30.5 & & 22.9 & 24.3 & \\
\hline$\geq 12$ & 80.0 & 69.5 & & 77.1 & 75.7 & \\
\hline
\end{tabular}

RSCC, right-sided colon cancer; LSCC, left-sided colon cancer; MAC, mucinous adenocarcinoma; NMAC, non-mucinous adenocarcinoma; SEER, Surveillance, Epidemiology, and End Results; AJCC, American Joint Committee on Cancer

As shown in Table 2, MAC was more often present on the right side of the colon $(14.2 \%$ vs. $7.1 \%$, $P<0.001)$. Because of the large sample size, statistically significant imbalances were detected in all patient characteristics between the RSCC and LSCC groups (except year of diagnosis) and between the MAC and NMAC groups. However, absolute differences in the proportions were numerically small. Of note, compared to patients with LSCC, those with RSCC were markedly more likely to be female $(55.3 \%$ vs. $47.8 \%)$, non-Hispanic white $(71.2 \%$ vs. $65.7 \%)$, and older than 80 years of age ( $26.3 \%$ vs. $15.3 \%)$, and they had poorly or non-differentiated $(27.4 \%$ vs. $17.1 \%)$ tumors, stage II tumors ( $43.3 \%$ vs. $37.3 \%)$, and $\geq 12$ evaluated nodes $(80.0 \%$ vs. $69.5 \%)$. More patients with MAC had T4 disease (25.2\% vs. $18.4 \%)$ compared to patients with NMAC.

After inverse propensity score weighting, patient characteristics were well balanced between each pair of the four $\mathrm{S}-\mathrm{H}$ groups in all three stage subgroups (Supplementary Tables A-C).

\section{Prognostic impact of sidedness in stage II-IV subgroups}

After inverse propensity score weighting, the median OS was significantly better in LSCC than in RSCC in the stage IV subgroup (20.5 vs. 16.5 months; HR for RSCC, 1.15 [95\% CI, 1.11-1.19]; P < 0.001; Figure 2A). The weighted 3-year OS rates for RSCC and LSCC were numerically similar in the stage III (68.6\% and 69.9\%, respectively; Figure 2B) and stage II subgroups (81.3\% and $80.5 \%$, respectively; Figure 2C), although the differences were statistically significant due to the large sample size (stage III subgroup: HR for RSCC, 1.06 [95\% CI, 1.01-1.09]; P = 0.006; stage II subgroup: HR for RSCC, 0.93 ; $95 \% \mathrm{CI}$, 089-0.97; P = 0.006).

\section{Prognostic value of mucinous histology by stage}

The weighted OS was significantly worse for MAC than NMAC in the stage IV (median OS, 16.9 vs. 19.5 months; HR for MAC, 1.15 [95\% CI, 1.09-1.22]; $P$ $<0.001$; Figure 3A) and stage III subgroups (3-year OS, $67.0 \%$ vs. $71.5 \%$; HR for MAC, 1.13 [95\% CI, 1.07-1.19]; $P<0.001$; Figure 3B). In the stage II subgroup, the weighted 3-year OS rates were similar for MAC and NMAC $(81.0 \%$ and $80.8 \%$, respectively; HR for MAC, 1.01 [95\% CI, 0.88-1.16]; $P=0.853$; Figure 3C).

In the stage III subgroup with inverse propensity score weighting, the prognostic interaction between sidedness and histology was also statistically significant ( $P_{\text {interaction }}<0.001$; Figure 4B and Table 3). The prognosis for non-mucinous RSCC was worse than for non-mucinous LSCC (3-year OS: $69.0 \%$ vs. 73.9\%; HR, 1.14 [95\% CI, 1.10-1.19]; P < 0.001), whereas the prognosis for mucinous RSCC was similar to that for mucinous LSCC (3-year OS: 68.2\% vs. $65.6 \%$; HR, 0.96 [95\% CI, 0.85-1.09]; $\mathrm{P}=0.531$ ). The prognosis for MAC on the left-hand side was markedly worse than NMAC on this side (3-year OS: $65.6 \%$ vs. $73.9 \%$; HR, 1.23 [95\% CI, 1.11-1.38]; $\mathrm{P}<$ 0.001 ), whereas the survival rates for MAC and NMAC on the right-hand side were similar (3-year OS: $68.2 \%$ vs. $69.0 \%$; HR, 1.04 [95\% CI, $0.97-1.11] ; \mathrm{P}=$ $0.300)$. 
A

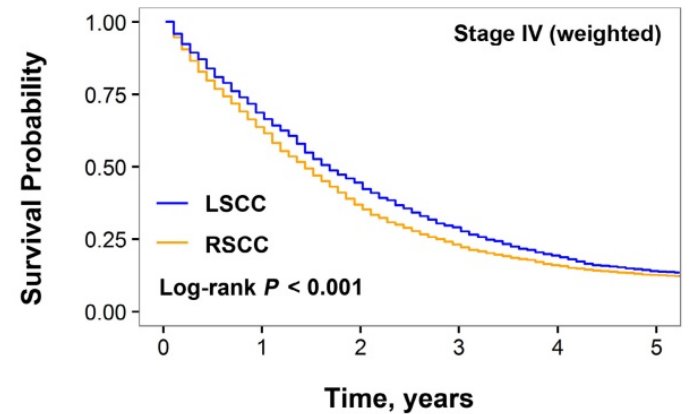

$\begin{array}{lllllll}\text { LSCC } & 8842 & 5052 & 2558 & 1387 & 780 & 474 \\ \text { RSCC } & 6606 & 4049 & 2291 & 1263 & 697 & 420\end{array}$

B

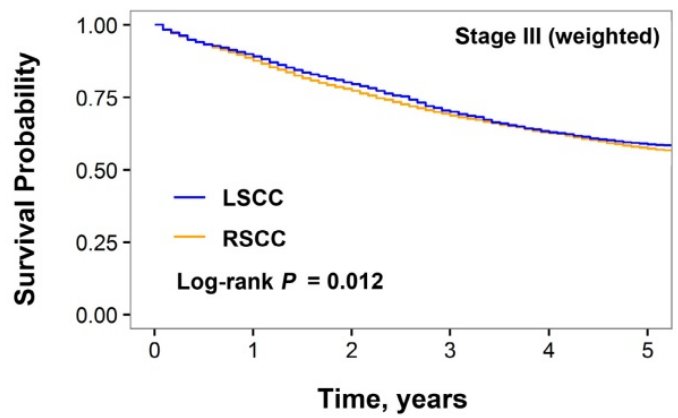

No. at risk

$\begin{array}{lllllll}\text { LSCC } & 19187 & 15135 & 11699 & 8821 & 6617 & 4726\end{array}$
RSCC
No. at risk

A

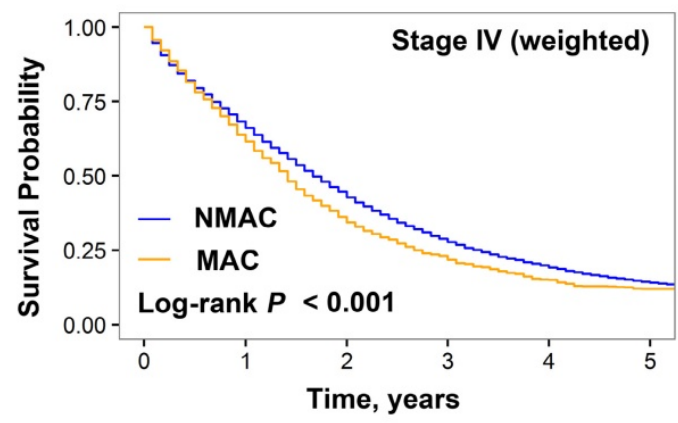

No. at risk

$\begin{array}{lcccccc}\text { NMAC } & 13710 & 8423 & 4819 & 2655 & 1514 & 862 \\ \text { MAC } & 1738 & 987 & 490 & 265 & 143 & 94\end{array}$

B

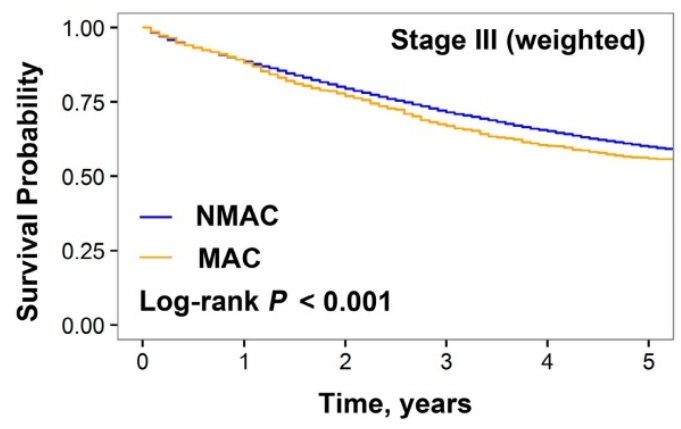

No. at risk

$\begin{array}{lllllll}\text { NMAC } & 29007 & 22948 & 18030 & 13912 & 10409 & 7476 \\ \text { MAC } & 3473 & 2758 & 2132 & 1568 & 1180 & 867\end{array}$

C

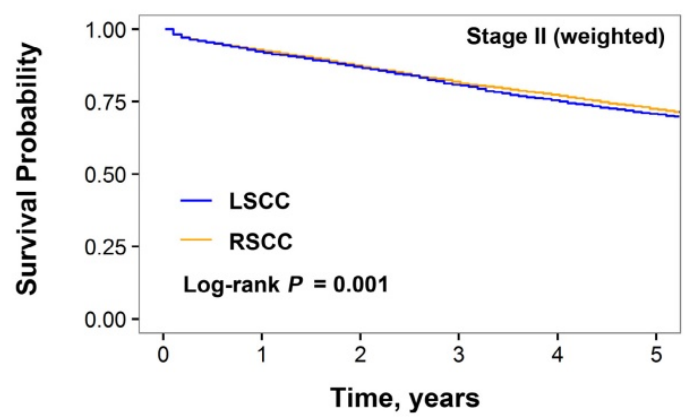

No. at risk

$\begin{array}{lllllll}\text { LSCC } & 21369 & 17603 & 14596 & 11708 & 9202 & 6743 \\ \text { RSCC } & 12045 & 9862 & 8214 & 6434 & 5016 & 3664\end{array}$

Figure 2. Kaplan-Meier estimates of overall survival of patients with right-sided colon cancer (RSCC) and left-sided colon cancer (LSCC). (A) Stage IV, (B) III, and (C) II subgroups weighted by inverse propensity score.

In the stage II subgroup, the weighted 3-year OS rates were similar between the non-mucinous RSCC, non-mucinous LSCC, mucinous RSCC, and mucinous LSCC groups $(81.0 \%, 80.6 \%, 81.6 \%$, and $80.5 \%$, respectively; $P=0.159$; Figure $4 \mathrm{C}$ and Table 3 ). Additionally, the interaction between tumor side and histology was insignificant $\left(P_{\text {interaction }}=0.466\right)$.

\section{Prognostic interaction between tumor side and histology}

In the stage IV subgroup weighted by inverse propensity score, the impact of sidedness on survival significantly differed according to tumor histology, and vice versa (test for interaction between sidedness and histology, $P_{\text {interaction }}<0.001$; Figure $4 \mathrm{~A}$ and Table 3). Patients with non-mucinous RSCC showed an 
A

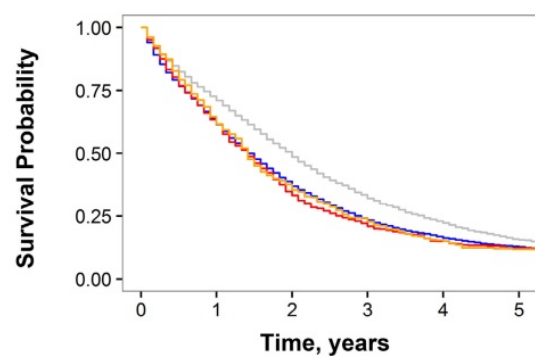

No. at risk

$\begin{array}{lcccccc}\text { Non-mucinous RSCC } & 7624 & 4368 & 2314 & 1235 & 712 & 416 \\ \text { Non-mucinous LSCC } & 6086 & 3991 & 2432 & 1372 & 776 & 432 \\ \text { Mucinous RSCC } & 1218 & 693 & 334 & 184 & 103 & 69 \\ \text { Mucinous LSCC } & 520 & 294 & 150 & 80 & 42 & 29\end{array}$

B

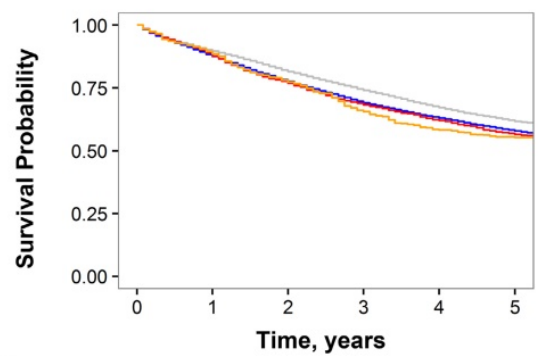

No. at risk

$\begin{array}{lcccccc}\text { Non-mucinous RSCC } & 16545 & 12960 & 10045 & 7639 & 5695 & 4094 \\ \text { Non-mucinous LSCC } & 12462 & 9956 & 7926 & 6201 & 4655 & 3340 \\ \text { Mucinous RSCC } & 2642 & 2099 & 1618 & 1209 & 913 & 647 \\ \text { Mucinous LSCC } & 831 & 659 & 511 & 370 & 277 & 211\end{array}$

\section{C}

No. at risk

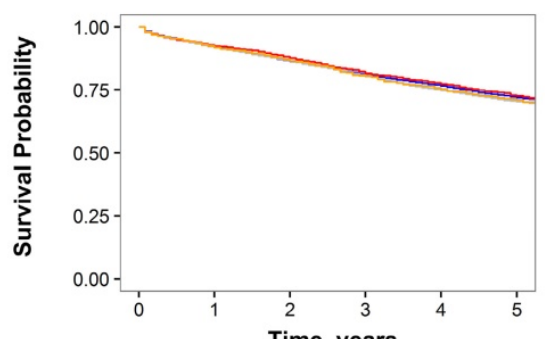

$\begin{array}{lllllll}\text { Non-mucinous RSCC } & 18226 & 15060 & 12352 & 9892 & 7739 & 5748\end{array}$

$\begin{array}{llllllll}\text { Non-mucinous LSCC } & 11134 & 9064 & 7470 & 5990 & 4570 & 3403\end{array}$

$\begin{array}{lllllll}\text { Mucinous RSCC } & 3143 & 2580 & 2163 & 1738 & 1372 & 992\end{array}$

$\begin{array}{lllllll}\text { Mucinous LSCC } & 911 & 750 & 631 & 482 & 385 & 275\end{array}$
Stage IV (weighted)

- Non-mucinous RSCC

- Non-mucinous LSCC

- Mucinous RSCC

- Mucinous LSCC

Log-rank $P<0.001$
Stage III (weighted)

- Non-mucinous RSCC

- Non-mucinous LSCC

- Mucinous RSCC

- Mucinous LSCC

Log-rank $P<0.001$

Figure 4. Kaplan-Meier estimates of overall survival for patients with non-mucinous right-sided colon cancer (RSCC), non-mucinous left-sided colon cancer (LSCC), mucinous RSCC, and mucinous LSCC. (A) Stage IV, (B) III, and (C) II subgroups weighted by inverse propensity score.

8-month decrease in median OS compared to those with non-mucinous LSCC (16.5 vs. 24.5 months; HR, 1.26 [95\% CI, 1.21-1.31]; P < 0.001), whereas patient survival was similar for mucinous RSCC and mucinous LSCC (median OS: 16.5 months for both; HR for mucinous RSCC, 1.03 [95\% CI, 0.92-1.16]; P = 0.570). Additionally, mucinous LSCC demonstrated an 8-month decrease in median OS compared with non-mucinous LSCC (16.5 vs. 24.5 months; HR, 1.27 [95\% CI, 1.14-1.41]; $\mathrm{P}<0.001)$, whereas patient survival was similar for mucinous RSCC and non-mucinous RSCC (median OS: 16.5 months for both; HR for mucinous RSCC, 1.05 [95\% CI, 0.97-1.12]; $\mathrm{P}=0.207)$.

\section{Sensitivity analysis}

For sensitivity analysis, we repeated the primary analyses after excluding patients with transverse colon cancer (10536 cases) or re-classifying these patients into the LSCC group. Additionally, we repeated the analyses after excluding patients in the tails of the distribution of propensity scores. [27] 
Under these circumstances, the conclusions were consistent with those of the primary analyses (data not shown).

Table 3. Kaplan-Meier estimates of overall survival (OS) and hazard ratios weighted by inverse propensity score

\begin{tabular}{|c|c|c|c|c|c|}
\hline Variable & $N$ & 3-y OS (\%) & $\begin{array}{l}\text { Median OS } \\
\text { (months) }\end{array}$ & $\begin{array}{l}\text { Hazard ratio } \\
(95 \% \text { CI })\end{array}$ & $P$ value \\
\hline Stage IV subgroup & 15448 & & & & \\
\hline \multicolumn{6}{|l|}{ Tumor side } \\
\hline Non-mucinous LSCC & 6086 & 32.1 & 24.5 & 1 & \\
\hline Non-mucinous RSCC & 7624 & 23.3 & 16.5 & $1.26(1.21-1.31)$ & $<0.001$ \\
\hline Mucinous LSCC & 520 & 22.8 & 16.5 & 1 & \\
\hline Mucinous RSCC & 1218 & 20.8 & 16.5 & $1.03(0.92-1.16)$ & 0.570 \\
\hline \multicolumn{6}{|l|}{ Mucinous histology } \\
\hline Non-mucinous LSCC & 6086 & 32.1 & 24.5 & 1 & \\
\hline Mucinous LSCC & 520 & 22.8 & 16.5 & $1.27(1.14-1.41)$ & $<0.001$ \\
\hline Non-mucinous RSCC & 7624 & 23.3 & 16.5 & 1 & \\
\hline Mucinous RSCC & 1218 & 20.8 & 16.5 & $1.05(0.97-1.12)$ & 0.207 \\
\hline Stage III subgroup & 32480 & & & & \\
\hline Tumor side & & & & & 0.012 \\
\hline Non-mucinous LSCC & 12462 & 73.9 & 99.5 & 1 & \\
\hline Non-mucinous RSCC & 16545 & 69.0 & 87.5 & $1.14(1.10-1.19)$ & $<0.001$ \\
\hline Mucinous LSCC & 831 & 65.6 & 79.5 & 1 & \\
\hline Mucinous RSCC & 2642 & 68.2 & 83.0 & $0.96(0.85-1.09)$ & 0.531 \\
\hline \multicolumn{6}{|l|}{ Mucinous histology } \\
\hline Non-mucinous LSCC & 12462 & 73.9 & 99.5 & 1 & \\
\hline Mucinous LSCC & 831 & 65.6 & 79.5 & $1.23(1.11-1.38)$ & $<0.001$ \\
\hline Non-mucinous RSCC & 2642 & 69.0 & 87.5 & 1 & \\
\hline Mucinous RSCC & 16545 & 68.2 & 83.0 & $1.04(0.97-1.11)$ & 0.300 \\
\hline \multicolumn{6}{|l|}{ Stage II subgroup } \\
\hline \multicolumn{6}{|l|}{ Tumor side } \\
\hline Non-mucinous LSCC & 11134 & 80.6 & Not reached & 1 & \\
\hline Non-mucinous RSCC & 18226 & 81.0 & Not reached & $0.96(0.91-1.00)$ & 0.072 \\
\hline Mucinous LSCC & 911 & 80.5 & Not reached & 1 & \\
\hline Mucinous RSCC & 3143 & 81.6 & Not reached & $0.90(0.78-1.05)$ & 0.176 \\
\hline \multicolumn{6}{|l|}{ Mucinous histology } \\
\hline Non-mucinous LSCC & 11134 & 80.6 & Not reached & 1 & \\
\hline Mucinous LSCC & 911 & 80.5 & Not reached & $1.04(0.91-1.19)$ & 0.540 \\
\hline Non-mucinous RSCC & 18226 & 81.0 & Not reached & 1 & \\
\hline Mucinous RSCC & 3143 & 81.6 & Not reached & $0.98(0.91-1.06)$ & 0.692 \\
\hline
\end{tabular}

\section{Discussion}

To the best of our knowledge, this is the first study that demonstrates that the prognostic impacts of tumor side and mucinous histology are affected by each other in stage III-IV colon cancer; that is, the prognosis for non-mucinous LSCC was significantly better than that for non-mucinous RSCC and that for mucinous LSCC, whereas the survival was similar between left-sided and right-sided MAC, and between mucinous and non-mucinous RSCC. In contrast, the survival was comparable among the four S-H subgroups for stage II colon cancer.

A number of previous studies have investigated the impact of sidedness and histology on survival outcomes for patients with colon cancer, reaching inconsistent conclusions. [3, 4, 6, 7, 11, 12, 19-21] None of these studies has taken into account sidedness, histology, and tumor stage simultaneously; to our knowledge, this is the first study to perform elaborate survival analyses with stratification for sidedness, histology, and tumor stage. The non-straightforward prognostic impact of sidedness and histology across different tumor stages, as well as the prognostic interaction between sidedness and histology for stage III/IV tumors, might partially explain the conflicting data in the previous studies.

Since clinicopathologic factors are optimally balanced by inverse propensity score weighting, tumor biology most probably drives the complex association of sidedness and histology with survival. Not only unfavorable molecular features (e.g., BRAF mutation, KRAS mutation, and $\mathrm{CpG}$ island methylation) but also favorable ones (e.g., deficient DNA mismatch repair [dMMR], chromosome stability and diploid) are more common in RSCC and MAC. [8, 9, 16, 28-32] As shown previously, the overall percentage of dMMR was up to $22 \%$ in stage II colon cancer, [33] which could partially balance out the negative prognostic impact of right sidedness and mucinous histology in the stage II subgroup. Moreover, the efficacy of chemotherapy and biologic agents may differ according to tumor side and histology, particularly in stage IV colon cancer. In the post hoc analysis of the FIRE1 trial, LSCC showed greater benefit from first-line cytotoxic chemotherapy than RSCC. [34] Additionally, reports of subgroup analysis from the FIRE3 and CALGB/SWOG 80405 trials suggest that patients with RSCC benefit less from anti-EGFR therapy than those with LSCC. [3, 35] Moreover, it was reported that MAC showed a poorer response to cytotoxic chemotherapeutic regimens than NMAC. [15, 16, 36] In the present study, the presence of either right sidedness or mucinous histology was correlated with an 8-month decrease in median OS and an over $25 \%$ increase in risk of death as compared with non-mucinous left-sided tumors in the stage IV subgroup, and the prognoses were similarly poor for stage IV non-mucinous RSCC, mucinous RSCC, and mucinous LSCC (16.5 months for all). Taken together, these findings suggest that both sidedness and histology should be considered during clinical decision-making processes and adopted as risk-stratification factors for advanced colon cancer. Moreover, recent studies suggest that despite dismal prognosis, the Commonsense Molecular Subtype 1 (predominantly right-sided tumors) and MAC are more likely to have a higher mutation count and stronger immune infiltration and activation.[2, 37] Therefore, further researches are warranted to investigate whether right-sidedness and/or mucinous histology can be used as additional markers to dMMR or high microsatellite instability for selecting candidates for PD-1 blockade. [38, 39] 
The therapeutic value of adjuvant chemotherapy in stage II colon cancer remains controversial, highlighting the need for effective risk-stratifying factors. Previous studies showed that the efficacy of adjuvant chemotherapy did not differ between stage II RSCC and LSCC or between stage II MAC and NMAC. [17, 19] Because of lack of evidence, current guidelines do not include right-sidedness and mucinous histology in the risk factor list for decision-making regarding adjuvant chemotherapy. [40] In the current study, the survival was similar in the four S-H groups for stage II colon cancer, which supports the current recommendation that consider neither of these factors when evaluating the administration of adjuvant treatment for stage II colon cancer.

The present study has some limitations. First, only limited data on patient-level characteristics have been provided by the SEER database, whereas data on patient comorbidities and performance status, tumor molecular features, and chemotherapy and chemotherapeutic regimens are unavailable. Because we assessed OS as the endpoint, medical comorbidities or other competing causes of death might influence our results. Still, OS is the most valuable endpoint for cancer patients and has no difference in definition between hospitals. Moreover, although the definition of the mucinous histology has been standardized, there might be variations in the diagnosis depending on the interpretation of the individual pathologist, which might result in misclassification. Despite these limitations, given the large sample size and the population-based nature of the SEER database, we are able to perform adequately powered survival analyses stratified by tumor side, histology, and stage, which is hard to achieve with single-institution studies.

\section{Conclusion}

In conclusion, the prognostic values of right sidedness and mucinous histology were mutually influenced for stage III and IV colon cancer. In contrast, the impacts of sidedness and histology on survival were minimal and independent of each other for stage II colon cancer. Thus, we recommend including both sidedness and histology as risk-stratification factors for patients with stage III/IV colon cancer but neither for those with stage II colon cancer.

\section{Acknowledgements}

We feel grateful that this work was supported by the Natural Science Foundation of Guangdong Province [grant number 2014A030312015]; Science and Technology Program of Guangdong [grant number 2015B020232008]; Science and Technology Program of
Guangzhou [grant number 15570006, 201508020250, 201604020003]. We also thank the staff members of the National Cancer Institute and their colleagues across the United States and at Information Management Services, Inc., who have been involved with the Surveillance, Epidemiology, and End Results (SEER) Program.

\section{Competing Interests}

The authors have declared that no competing interest exists.

\section{References}

1. Torre LA, Bray F, Siegel RL, Ferlay J, Lortet-Tieulent J, Jemal A. Global cancer statistics, 2012. CA Cancer J Clin. 2015; 65: 87-108.

2. Guinney J, Dienstmann R, Wang X, De RA, Schlicker A, Soneson C, et al. The Consensus Molecular Subtypes of Colorectal Cancer. Nat Med. 2015; 21: 1350-6.

3. Venook A ND, Innocenti $F$ et al. Impact of primary $\left(1^{\circ}\right)$ tumor location on overall survival (OS) and progression-free survival (PFS) in patients (pts) with metastatic colorectal cancer (mCRC): Analysis of CALGB/SWOG 80405 (Alliance). ASCO Meeting Abstracts. 2016; 34: 3504.

4. Warschkow R, Tarantino I, Huttner FJ, Schmied BM, Guller U, Diener MK, et al. Predictive value of mucinous histology in colon cancer: a population-based, propensity score matched analysis. Br J Cancer. 2016; 114: 1027-32.

5. Saltzstein SL, Behling CA. Age and time as factors in the left-to-right shift of the subsite of colorectal adenocarcinoma: a study of 213,383 cases from the California Cancer Registry. J Clin Gastroenterol. 2007; 41: 173-7.

6. Warschkow R, Sulz MC, Marti L, Tarantino I, Schmied BM, Cerny T, et al. Better survival in right-sided versus left-sided stage I - III colon cancer patients. BMC Cancer. 2016; 16: 554

7. Weiss JM, Pfau PR, O'Connor ES, King J, LoConte N, Kennedy G, et al. Mortality by stage for right- versus left-sided colon cancer: analysis of surveillance, epidemiology, and end results--Medicare data. J Clin Oncol. 2011; 29: 4401-9.

8. Missiaglia E, Jacobs B, D'Ario G, Di Narzo AF, Soneson C, Budinska E, et al. Distal and proximal colon cancers differ in terms of molecular, pathological, and clinical features. Ann Oncol. 2014; 25: 1995-2001.

9. Loupakis F, Yang D, Yau L, Feng S, Cremolini C, Zhang W, et al. Primary Tumor Location as a Prognostic Factor in Metastatic Colorectal Cancer. JNCI Journal of the National Cancer Institute. 2015; 107: dju427-dju.

10. Sinicrope FA, Shi Q, Smyrk TC, Thibodeau SN, Dienstmann R, Guinney J, et al. Molecular markers identify subtypes of stage III colon cancer associated with patient outcomes. Gastroenterology. 2015; 148: 88-99.

11. Meguid RA, Slidell MB, Wolfgang CL, Chang DC, Ahuja N. Is there a difference in survival between right- versus left-sided colon cancers? Ann Surg Oncol. 2008; 15: 2388-94.

12. Schrag D WS, Brooks G et al. The relationship between primary tumor sidedness and prognosis in colorectal cancer. ASCO Meeting Abstracts. 2016; 34: 3505.

13. Jass JR, Sobin LH. Histological Classification of Intestinal Tumours. Berlin, Heidelberg: Springer; 1989

14. Chiang JM, Yeh CY, Changchien CR, Chen JS, Tang R, Chen JR. Mucinous adenocarcinoma showing different clinicopathological and molecular characteristics in relation to different colorectal cancer subgroups. Int J Colorectal Dis. 2010; 25: 941-7.

15. Negri FV, Wotherspoon A, Cunningham D, Norman AR, Chong G, Ross PJ. Mucinous histology predicts for reduced fluorouracil responsiveness and survival in advanced colorectal cancer. Ann Oncol. 2005; 16: 1305-10.

16. Mekenkamp LJ, Heesterbeek KJ, Koopman M, Tol J, Teerenstra S, Venderbosch S, et al. Mucinous adenocarcinomas: poor prognosis in metastatic colorectal cancer. Eur J Cancer. 2012; 48: 501-9.

17. Verhulst J, Ferdinande L, Demetter P, Ceelen W. Mucinous subtype as prognostic factor in colorectal cancer: a systematic review and meta-analysis. J Clin Pathol. 2012; 65: 381-8.

18. Kim SH, Shin SJ, Lee KY, Kim H, Kim TI, Kang DR, et al. Prognostic value of mucinous histology depends on microsatellite instability status in patients with stage III colon cancer treated with adjuvant FOLFOX chemotherapy: a retrospective cohort study. Ann Surg Oncol. 2013; 20: 3407-13.

19. Catalano V, Loupakis F, Graziano F, Bisonni R, Torresi U, Vincenzi B, et al. Prognosis of mucinous histology for patients with radically resected stage II and III colon cancer. Ann Oncol. 2012; 23: 135-41.

20. Hugen N, Verhoeven RH, Radema SA, de Hingh IH, Pruijt JF, Nagtegaal ID, et al. Prognosis and value of adjuvant chemotherapy in stage III mucinous colorectal carcinoma. Ann Oncol. 2013; 24: 2819-24.

21. Hogan J, Burke JP, Samaha G, Condon E, Waldron D, Faul P, et al. Overall survival is improved in mucinous adenocarcinoma of the colon. Int J Colorectal Dis. 2014; 29: 563-9. 
22. McCaffrey DF, Griffin BA, Almirall D, Slaughter ME, Ramchand R, Burgette LF. A tutorial on propensity score estimation for multiple treatments using generalized boosted models. Stat Med. 2013; 32: 3388-414.

23. Dehejia RH, Wahba S. Propensity score-matching methods for nonexperimental causal studies. Rev Econ Stat. 2002; 84: 151-61.

24. Austin PC. The performance of different propensity score methods for estimating marginal hazard ratios. Stat Med. 2013; 32: 2837-49.

25. Wang Y, Cai H, Li C, Jiang Z, Wang L, Song J, et al. Optimal caliper width for propensity score matching of three treatment groups: a Monte Carlo study. PLoS One. 2013; 8: e81045.

26. Lin DY, Wei LJ. The robust inference for the Cox proportional hazards model. J Am Stat Assoc. 1989; 84: 1074-8.

27. Stürmer T, Rothman KJ, Avorn J, Glynn RJ. Treatment effects in the presence of unmeasured confounding: dealing with observations in the tails of the propensity score distribution - a simulation study. Am J Epidemiol. 2010; 172: 843-54.

28. Pai RK, Jayachandran P, Koong AC, Chang DT, Kwok S, Ma L, et al. BRAF-mutated, microsatellite-stable adenocarcinoma of the proximal colon: an aggressive adenocarcinoma with poor survival, mucinous differentiation, and adverse morphologic features. Am J Surg Pathol. 2012; 36: 744-52.

29. Tran B, Kopetz S, Tie J, Gibbs P, Jiang ZQ, Lieu CH, et al. Impact of BRAF mutation and microsatellite instability on the pattern of metastatic spread and prognosis in metastatic colorectal cancer. Cancer. 2011; 117: 4623-32.

30. Hutchins G, Southward K, Handley K, Magill L, Beaumont C, Stahlschmidt I, et al. Value of mismatch repair, KRAS, and BRAF mutations in predicting recurrence and benefits from chemotherapy in colorectal cancer. J Clin Oncol. 2011; 29: 1261-70.

31. Ogino S, Brahmandam M, Cantor M, Namgyal C, Kawasaki T, Kirkner G, et al. Distinct molecular features of colorectal carcinoma with signet ring cell component and colorectal carcinoma with mucinous component. Mod Pathol. 2006; 19: 59-68.

32. Tanaka H, Deng G, Matsuzaki K, Kakar S, Kim GE, Miura S, et al. BRAF mutation, $\mathrm{CpG}$ island methylator phenotype and microsatellite instability occur more frequently and concordantly in mucinous than non-mucinous colorectal cancer. Int J Cancer. 2006; 118: 2765-71.

33. Roth AD, Tejpar S, Delorenzi M, Yan P, Fiocca R, Klingbiel D, et al. Prognostic role of KRAS and BRAF in stage II and III resected colon cancer: results of the translational study on the PETACC-3, EORTC 40993, SAKK 60-00 trial. J Clin Oncol. 2010; 28: 466-74

34. Modest DP, Schulz C, von Weikersthal LF, Quietzsch D, von Einem JC, Schalhorn A, et al. Outcome of patients with metastatic colorectal cancer depends on the primary tumor site (midgut vs. hindgut): analysis of the FIRE1-trial (FuFIRI or mIROX as first-line treatment). Anticancer Drugs. 2014; 25: $212-8$

35. Heinemann V MD, Fischer von Weikersthal L et al. Gender and tumor location as predictors for efficacy: influence on endpoints in first-line treatment with FOLFIRI in combination with cetuximab or bevacizumab in the AIO KRK 0306 (FIRE3) trial. ASCO Meeting Abstracts. 2014; 32: 3600.

36. Catalano V, Loupakis F, Graziano F, Torresi U, Bisonni R, Mari D, et al. Mucinous histology predicts for poor response rate and overall survival of patients with colorectal cancer and treated with first-line oxaliplatin- and/or irinotecan-based chemotherapy. Br J Cancer. 2009; 100: 881-7.

37. Shia J, Schultz N, Kuk D, Vakiani E, Middha S, Segal NH, et al. Morphological characterization of colorectal cancers in The Cancer Genome Atlas reveals distinct morphology-molecular associations: clinical and biological implications. Mod Pathol. 2017; 30: 599-609.

38. Le DT, Uram JN, Wang H, Bartlett BR, Kemberling H, Eyring AD, et al. PD-1 Blockade in Tumors with Mismatch-Repair Deficiency. N Engl J Med. 2015; 372: 2509-20.

39. Le DT, Durham JN, Smith KN, Wang H, Bartlett BR, Aulakh LK, et al. Mismatch-repair deficiency predicts response of solid tumors to PD-1 blockade. Science (New York, NY). 2017; 357: 409-13.

40. [Internet] National Comprehensive Cancer Network (NCCN). NCCN clinical practice guidelines in oncology (NCCN guidelines): colon cancer. Accessed 17 July 2017. http://www.nccn.org/professionals/physician_gls/f_guidelines_ nojava.asp. 